\title{
Selected momentum correction using induction-voltage modulator for low-flux ion beams
}

\author{
Takashi Kikuchi* \\ Department of Electrical and Electronic Engineering, Utsunomiya University, \\ Yohtoh 7-1-2, Utsunomiya 321-8585, Japan \\ Takeshi Katayama \\ Center for Nuclear Study, Graduate School of Science, University of Tokyo, \\ Wako-Branch at RIKEN, 2-1 Hirosawa, Wako, Saitama, 351-0198, Japan \\ Kazuhiko Horioka \\ Department of Energy Sciences, Interdisciplinary Graduate School of Science and Engineering, Tokyo Institute of Technology, \\ Nagatsuta 4259, Midori-ku, Yokohama, 226-8502, Japan \\ Shigeo Kawata \\ Department of Energy and Environmental Science, Utsunomiya University, Yohtoh 7-1-2, Utsunomiya 321-8585, Japan
}

(Received 10 November 2004; published 30 August 2005)

\begin{abstract}
A method for direct momentum correction of a selected beam particle using a controllable inductionvoltage modulator is proposed for a low-flux ion beam. The corrected ion beam has a small momentum error restricted by a detection error at a kinetic energy analyzer and a voltage fluctuation at the inductionvoltage modulator. Numerical simulations indicate that the scheme using a multiturn correction in a storage ring can considerably reduce the requirement of the amplitude and the tolerance level of the correction voltage. The results also show the final momentum error of the order of $10^{-5}$ can be rapidly achieved at total flight time of $30 \mu$ s when repetitive correction voltages are applied during the revolutions in the ring.
\end{abstract}

DOI: 10.1103/PhysRevSTAB.8.080402

PACS numbers: 29.27.Eg, 29.27.Ac

\section{INTRODUCTION}

Radioactive isotope (RI) beams [1] are a crucial tool for developing future studies on reactions and properties of unstable nuclei. Many facilities involving the use of RI beams are operated, being upgraded, and under constructions in the world [2]. At the GSI (Darmstadt, Germany), a storage ring for RI beams was developed, and mass spectroscopy experiments were carried out [3-6]. At the RIKEN facility, RI-beam factory, which is under construction, can produce many ion species of rare and unstable RI beams [7]. RI beams produced by projectile fragmentation in heavy ion collision are collected by RI-beam separators [8]. It is predicted that typical production rates of the secondary beams are low as 1 to $1000 \mathrm{~s}^{-1}$, so that the RI beam must be efficiently utilized for experiments, such as mass measurements and internal targets [4]. The quality of RI beam is the most important parameter for the injection into the storage ring and for the guidance to the experimental apparatuses. The RI-beam separators have a rather large acceptance in the transverse plane and a momentum spread for the rare RI collection. Since the storage ring and the experimental setup have a finite acceptance, the injection and guidance of the RI beam with a large transverse emittance and a large momentum spread must be regulated.

*Electronic address: tkikuchi@cc.utsunomiya-u.ac.jp
For the improvement of transverse emittance, an individual trajectory correction scheme was proposed [9-11]. The parameters of each RI are measured individually, and the particle trajectories can be corrected by a fast kicker system. The momentum spread of the particles is reduced using a profiled energy degrader [9-11]. However, the quality of the momentum correction by the degrader depends on the betatron motion of the RIs, the nonzero dispersion, the multiple scattering, and the energy straggling in the degrader section. After the corrections, the RI beam is injected into the storage ring by an individual injection method [9-11]. In the method, the injection kicker should be operated to synchronize with the new injected RI and the stack particles in the ring. Providing the fast stacking of the injected RI, several options using beam cooling were proposed [9-12]. One of the methods is to use an electron cooling system with an induction betatron accelerator, which can compensate the initial momentum deviation of the injected RI. The induction accelerator can be used to push the RIs into the optimal region of the electron cooling force [13]. Even with the improved electron cooling system mentioned above and a stochastic cooling [12], the cooling time is still the order of several $10 \mathrm{~ms}$ [10-12].

An induction-voltage modulator [14,15], which is based on a voltage adder configuration, can make arbitrary waveform. Recently, induction accelerator technology has been applied to synchrotron $[16,17]$. The induction-voltage 
module, which has a precise waveform controllability and a high repetition rate, is developed [18,19]. Since the operation of the induction-voltage modulator is based on solid-state power devices, we can apply the voltage to a charged particle beam in a controllable manner.

We propose a selected momentum correction method of each charged particle using the induction-voltage modulator. When a momentum error from the destination energy of the selected particle is detected, the induction-voltage modulator can individually kick the particle for the momentum correction, directly. The repetitive operation of the method brings the rapid reduction of the momentum error. By using the proposed scheme, the momentum error of each charged particle is corrected, and we will be able to use the cooled beam for the above purposes.

\section{SELECTED MOMENTUM CORRECTION METHOD}

Figure 1 shows the outline of this scheme. The particle energy can be detected by an analyzer at the entrance of beam transport line. The discrepancy of particle energy from the destination value is obtained by the information from the analyzer. For a compensation of the detected energy difference, the induction modulator can apply the correction voltage. Consequently, the particle energy is corrected one by one to the destination energy, and the momentum error approaches to zero, rapidly. By using the proposed scheme, we can control the longitudinal momentum of the beam particle, efficiently. This selected momentum correction scheme together with the individual trajectory correction also provides us a novel method of fast beam cooling.

The voltage applied by the induction modulator can regulate each particle energy. The relation between the corrected particle energy $E_{c}$ and the applied voltage $V$ is

$$
E_{c}=E_{i}+q V
$$

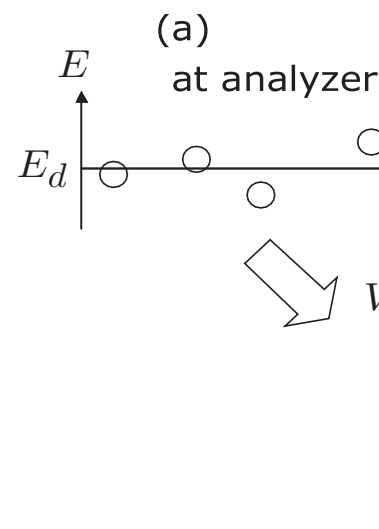

(b) at Induction Modulator where $E_{i}$ is the initial particle energy before the gap of induction unit and $q$ is the charge state of the particle. The applied voltage is assumed as $V=V_{\text {ind }}+\Delta V_{f}$, where $V_{\text {ind }}$ is the voltage from the induction modulator and $\Delta V_{f}$ is the fluctuation of voltage applied by the induction module, respectively.

Using the analyzer based on the time-of-flight (TOF) method [20], we are able to determine the particle energy with a somewhat high accuracy. However the analyzer has an error from the true value of the particle energy. As a result, the analyzed particle energy $E_{a}$ is given as $E_{a}=$ $E_{i}+\Delta E_{a}$, where $\Delta E_{a}$ is the error at the analyzer.

The voltage required at the induction modulator is determined by

$$
q V_{\text {ind }}=E_{d}-E_{a},
$$

where $E_{d}$ is the destination particle energy. Figure 2 shows the relations between the particle energy, the voltage applied by the induction module, and the destination energy. Here the voltage applied by the induction module is assumed as $V=V_{\text {ind }}+\Delta V_{f}<V_{\text {limit }}$, which is the voltage limitation of the induction module. As an ideal situation without any fluctuations and errors, the condition of $E_{d}=$ $E_{c}$ is satisfied, i.e., $q V_{\text {ind }}=E_{d}-E_{i}$. As shown in Fig. 3, the error at the analyzer is not important for $V=V_{\text {ind }}+$ $\Delta V_{f}>V_{\text {limit }}$ condition, because the voltage is restricted by the limitation value. In this case, the modulated particle energy is replaced by $E_{c}=E_{i}+q\left(V_{\text {ind }}+\Delta V_{f}\right)$, for $\mid E_{d}-$ $E_{a} \mid \gg q V_{\text {limit }}$. Under the above ideal condition, for the initial momentum error $(\Delta p / p)_{i}$, the voltage required at the induction module is estimated by

$$
\begin{aligned}
\left|\frac{q V_{\text {ind }}}{A}\right|= & e_{d}+m_{u} c^{2} \\
& -\sqrt{m_{u}^{2} c^{4}+e_{d}\left(e_{d}+2 m_{u} c^{2}\right)\left[\left(\frac{\Delta p}{p}\right)_{i}+1\right]^{2}},
\end{aligned}
$$

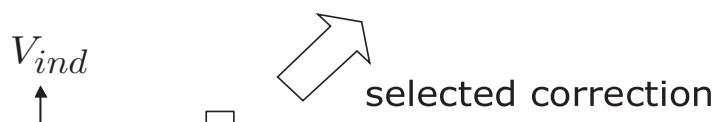

FIG. 1. Outline of selected momentum correction scheme. (a) At the analyzer the particle energy can be detected, (b) the induction modulator can apply the correction voltage, and (c) the particle energy is corrected one by one to the destination energy. 


\section{(a)}

at analyzer

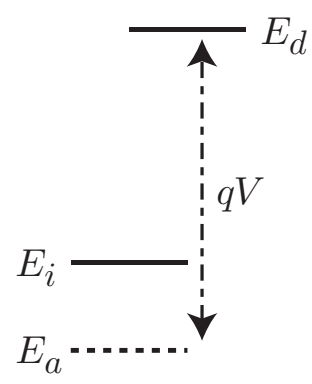

(b) after induction gaps

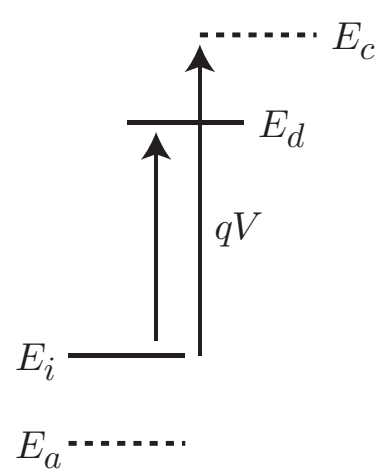

FIG. 2. Particle energy modulated by an induction-voltage modulator for $V=V_{\text {ind }}+\Delta V_{f}<V_{\text {limit }}$, (a) at the analyzer and (b) after the induction-voltage modulator.

where $A$ is the atomic mass number of the beam ion, $e_{d}=$ $E_{d} / A$ is the destination particle energy per nucleon, $m_{u}$ is the atomic mass unit, and $c$ is speed of light, respectively. Here the initial momentum error is defined by

$$
\left(\frac{\Delta p}{p}\right)_{i}=\left(\frac{2 m_{u} c^{2} e_{i}+e_{i}^{2}}{2 m_{u} c^{2} e_{d}+e_{d}^{2}}\right)^{1 / 2}-1
$$

where $e_{i}=E_{i} / A$ is the particle energy per nucleon before the correction. Figure 4 shows the estimated voltage required to the induction module for the momentum correction.

Substituting Eq. (2) to Eq. (1), the modulated particle energy is rewritten by $E_{c}=E_{d}-\Delta E_{a}+q \Delta V_{f}$. From $E_{c}=A e_{c}$ and $E_{d}=A e_{d}$, we normalize the equation as

$$
e_{c}=e_{d}-\frac{\Delta E_{a}}{E_{d}} e_{d}+\frac{q V_{\text {ind }}}{A} \frac{\Delta V_{f}}{V_{\text {ind }}} .
$$

As written in Eq. (5), the corrected particle energy is restricted by the voltage fluctuation of induction module

(a)

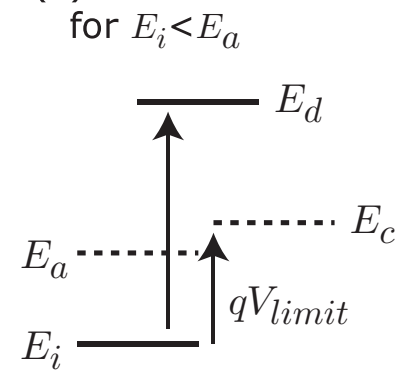

(b)

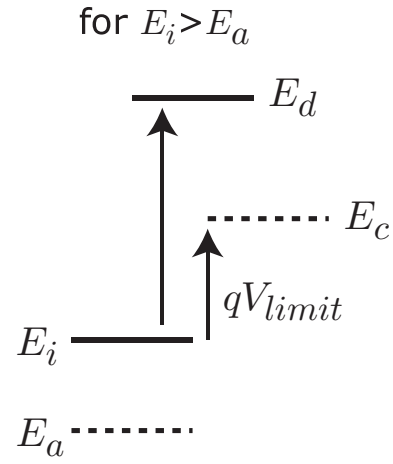

FIG. 3. Modulated particle energy by an induction-voltage modulator in case of $V=V_{\text {ind }}+\Delta V_{f}>V_{\text {limit }}$, (a) for $E<E_{a}$, (b) for $E>E_{a}$.

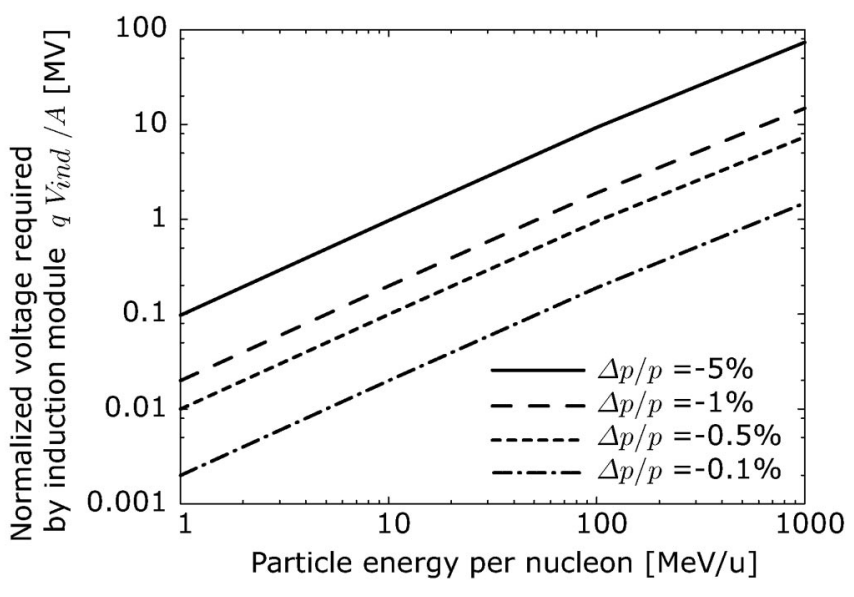

FIG. 4. Voltage required at an induction modulator as a function of particle energy per nucleon at each momentum error before the correction.

and the error given at the particle energy analyzer. The corrected momentum error is defined by

$$
\left(\frac{\Delta p}{p}\right)_{c}=\left(\frac{2 m_{u} c^{2} e_{c}+e_{c}^{2}}{2 m_{u} c^{2} e_{d}+e_{d}^{2}}\right)^{1 / 2}-1,
$$

where $e_{c}=E_{c} / A$ is the corrected particle energy per nucleon.

By using random numbers we calculate the momentum error after the correction. The random number simulates the error at the analyzer and the voltage fluctuation at the induction module. From Eq. (5), we can calculate the corrected particle energy including the detection errors and voltage fluctuations. By random numbers $r_{G 1 n}$ and $r_{G 2 n}$, we describe the analyzing error term as

$$
\frac{\Delta E_{a}}{E_{d}}=\left(\frac{\Delta E_{a}}{E_{d}}\right)_{0} r_{G 1 n}
$$

and the voltage fluctuation term as

$$
\frac{\Delta V_{f}}{V_{\text {ind }}}=\left(\frac{\Delta V_{f}}{V_{\text {ind }}}\right)_{0} r_{G 2 n},
$$

where $n$ means that it is the $n$th random number. It is assumed that the random numbers have a normal distribution. The Mersenne Twister method is used for the pseudorandom number generator [21], and the random numbers for the normal distribution consist of the uniform random number sets.

Figure 5 shows the rms value of the corrected momentum error versus the analyzing error and the voltage fluctuation. When the analyzing error ratio $\Delta E_{a} / E_{d}$ is large $(>0.1 \%)$, the corrected momentum error is independent of the amplitude of the voltage fluctuation. Figure 5 indicates that to obtain the corrected momentum error less than $10^{-5}$, the voltage fluctuation should be suppressed less than $0.1 \%$, when the analyzed error is less than $10^{-5}$. In practice, the voltage control at fluctuation less than $1 \%$ 


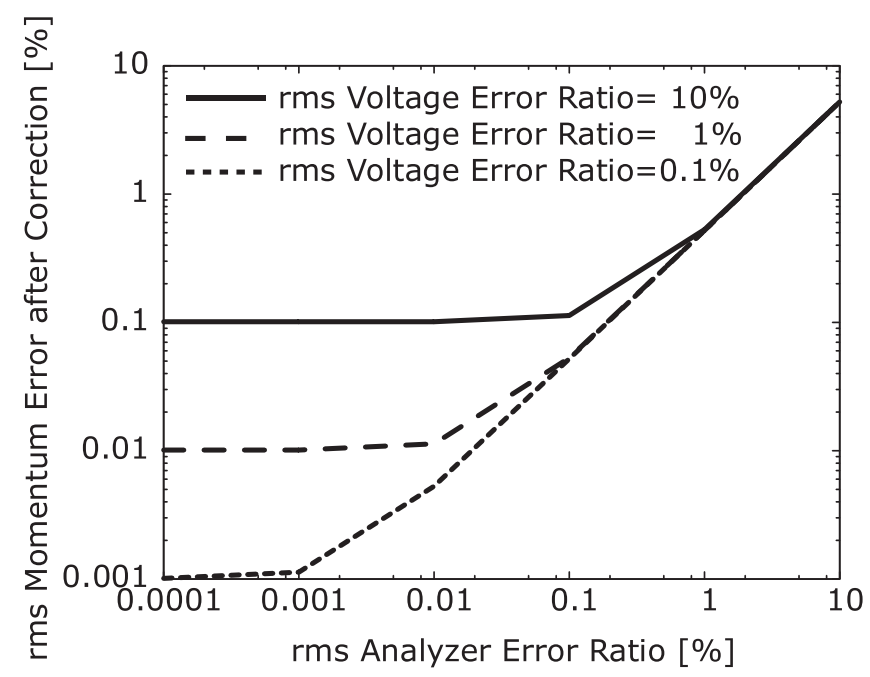

FIG. 5. Corrected momentum error as a function of detection errors at an analyzer and voltage fluctuations of an induction modulator. The particle energy per nucleon is assumed as $100 \mathrm{MeV} / \mathrm{u}$. The initial momentum error is $-1 \%$. The normalized voltage required $q V_{\text {ind }} / A$ is $1.9 \mathrm{MV}$ as shown in Fig. 4.

is difficult under the high voltage pulsed-power operation. The corrected momentum error is substantially limited by the voltage fluctuation at the induction module.

\section{REGULATION OF VOLTAGE APPLIED BY INDUCTION-VOLTAGE MODULATOR}

The correction voltage should be maintained during the particle transit in an acceleration gap. For this reason, the pulse duration $\tau_{p}$ for the induction voltage is given as $\tau_{p}=$ $L_{g} / v_{p}$, where $L_{g}$ is the length of the acceleration gaps in the induction module and $v_{p}$ is the particle speed. As a result, the pulse duration is roughly required as

$$
\tau_{p}=\frac{L_{g}\left(e_{i}+m_{u} c^{2}\right)}{c \sqrt{e_{i}\left(e_{i}+2 m_{u} c^{2}\right)}} .
$$

Figure 6 shows the pulse duration required by the induction-voltage modulator at various particle energies. Recently, an induction-voltage modulator, which can be operated at $\mathrm{MHz}$, is developed [18]. The repetition rate $R_{\text {ind }}\left(\mathrm{s}^{-1}\right)$ required by the induction-voltage modulator is given by

$$
R_{\mathrm{ind}}=\frac{1}{\tau_{p r}+\tau_{p}+\tau_{p f}+\tau_{r r}+\tau_{r}+\tau_{r f}},
$$

where $\tau_{p r}$ and $\tau_{p f}$ are the rise and fall times for the main pulse duration, $\tau_{r}$ is the pulse duration for the reset of induction core, $\tau_{r r}$ and $\tau_{r f}$ are the rise and fall times for the reset pulse, respectively. Figure 7 shows the typical waveform of induction voltage. In the induction accelerator technology, the magnetic core of the module must be reset

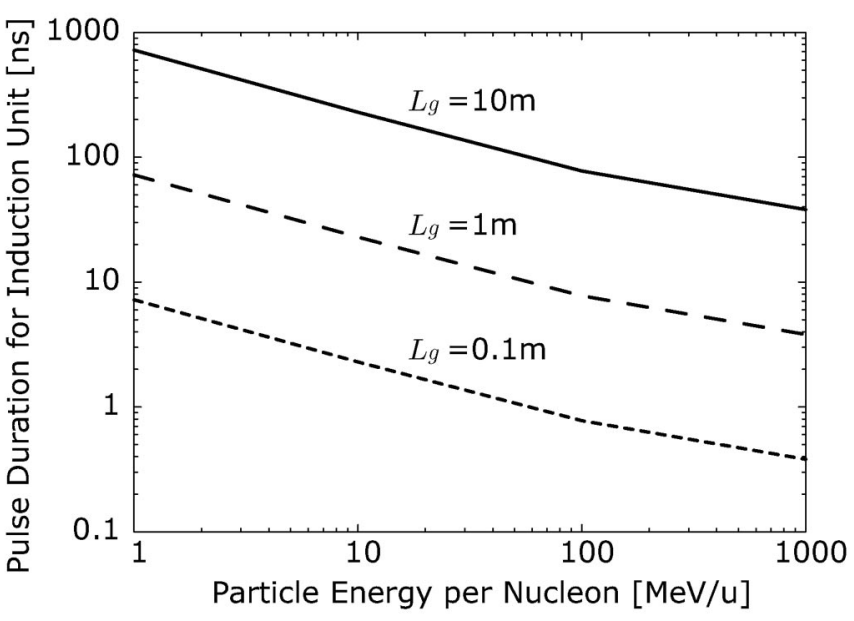

FIG. 6. Estimated pulse duration for an induction-voltage modulator as a function of particle energy per nucleon.

by the reversal voltage pulses. The voltage-time product offsets the value of the main pulse, i.e., $V_{p}\left(2 \tau_{p}+\tau_{p r}+\right.$ $\left.\tau_{p f}\right)=V_{r}\left(2 \tau_{r}+\tau_{r r}+\tau_{r f}\right)$. In the induction module, the relation of $V \tau=S \Delta B$ is one of the limitations for the operation, where $V$ is the voltage applied by the induction module, $\tau$ is the pulse duration, $S$ is the cross section area of the core, and $\Delta B$ is the maximum magnetic flux-swing in the core, respectively. Because of this limitation, the operation at long pulse duration restricts the allowable voltage of the induction module. In the field of heavy ion inertial fusion, the voltage-time product is required as 0.45 to $1.5 \mathrm{Vs}$ per meter $[22,23]$. From this point of view and Fig. 6, several MV voltage can be applied to the gaps of the induction module with a large core size. However precise voltage control at MV level with the high repetition rate is quite difficult.

The key technology of this proposed scheme is the voltage modulator, which must be operable at each timing with an arbitrary correction voltage. The induction-voltage modulator, which consists of the induction adder system with the solid-state semiconductor switch, is the candidate.

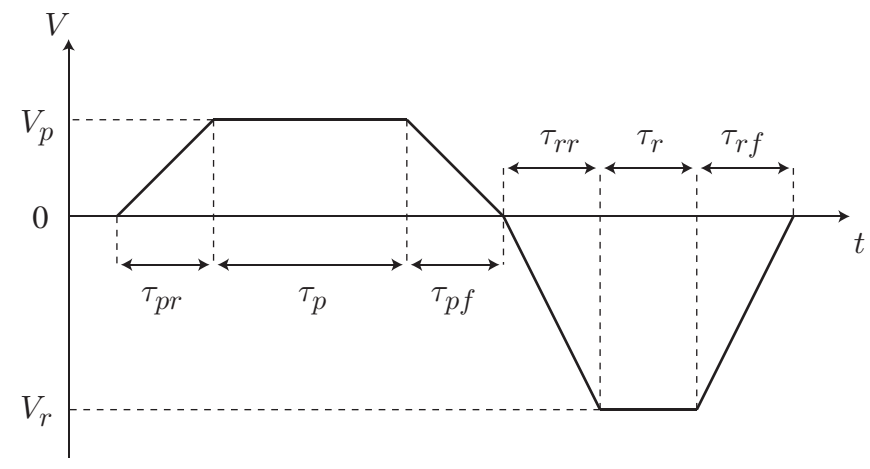

FIG. 7. Typical induction-voltage waveform. The waveform consists of main and reset pulses. 
The complex can be operated by control of the peak voltage at the beam arrival time. The correction voltages are prepared by combining the several electrically charged modules. When the amplitude of correction voltage is determined from the particle energy detected by the analyzer, the electrical charged modules can apply the correction voltages to the induction adder system by overlapping the voltages of induction cells.

\section{MULTITURN OPERATION IN A RING}

Although the solid-state semiconductor devices have good controllability for the trigger timing, the operation at the high voltage is difficult to manage and the overlapped voltage is predicted to have a random error. For this reason, the multiturn voltage additions in a storage ring may be required for the application of this proposed scheme.

Figure 8 shows the typical simulation result for the multiturn momentum correction. The limitation voltage assumed in the calculation is $100 \mathrm{kV}$, and the controlled voltages are applied to the particle at each lap. The initial momentum error is $-1 \%$ at the central particle energy of $100 \mathrm{MeV} / \mathrm{u}$ with $q / A=0.5$. The coefficient $\left(\Delta V_{f} / V_{\text {ind }}\right)_{0}$ of voltage applied by the induction modulator has the fluctuation of $5 \%$, and the detection error coefficient $\left(\Delta E_{a} / E_{d}\right)_{0}$ at the analyzer of the particle energy is assumed to $10^{-4}$. The ring circumference is $77.76 \mathrm{~m}$, and the revolution period of the beam is $0.6 \mu$ s. As shown in Fig. 8, the momentum error of the beam rapidly approaches to zero. The rms value of the final momentum error after 50 laps is $5.29 \times 10^{-5}$ for the calculation of 10000 test particles at the total flight time of $30 \mu \mathrm{s}$.

In the momentum correction with the multiturn feedback operations, the voltage required at the induction module reduces to $\left(E_{i}-E_{d}\right) / q N_{\text {lap }}$, so that an allowable voltage

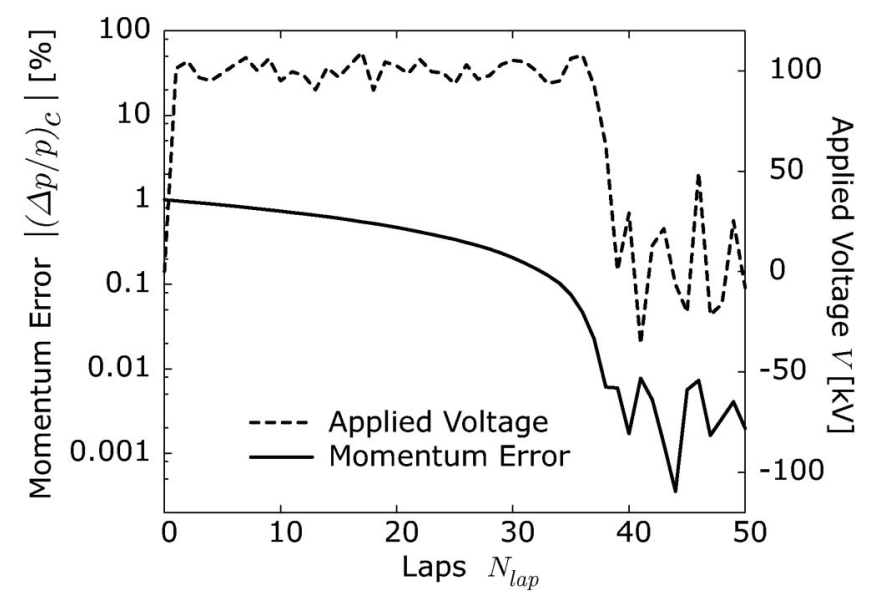

FIG. 8. Momentum error and voltage applied by induction module during multilaps in a ring. fluctuation increases by a factor of $N_{\text {lap }}$, where $N_{\text {lap }}$ denotes the revolution number.

\section{CONCLUSION}

In this paper, we proposed the selected momentum correction method for a single particle with low ion flux. The error of particle energy detected by the analyzer can be corrected by the voltage applied at the induction modulator. For a single path correction scheme, when the error at the analyzer and voltage fluctuations are small enough, e.g., $\Delta E_{a} / E_{d}=10^{-5}$ and $\Delta V_{f} / V_{\text {ind }}=10^{-3}$, the momentum error of $10^{-5}$ is achieved as shown in Fig. 5. In the case of the single path correction scheme, control of a high voltage modulator is a challenging problem.

The proposed scenario is much more advantageous with a multiturn operation in the storage ring, because the achievable final momentum error is smaller than the single path operation with the lower voltage required at the induction module. Also the accuracy of TOF can be increased with the lap number. In this in situ feedback multiturn correction scheme, the momentum error rapidly approaches to zero by the correction voltage.

For the longitudinal and transverse beam cooling, the momentum correction method can work in the combination with the transverse orbit correction scheme, such as the individual trajectory correction.

The storage rate and number of stacked RIs in an accumulator ring are important factors for the effective use of the generated rare RIs. A few injection schemes were investigated and were compared in Refs. [9-11] by Meshkov et al., for the rare RI accumulation. As mentioned in their papers, a new RI injection can interfere the storage of RIs stacked in the accumulator ring, so that the storage rate and number of the RIs in the ring are restricted. The parameters are also limited in the momentum correction method proposed in this paper. The accumulation of RIs in the storage ring with the use of this selected momentum correction method has a problem still remained as our future work. One of the methods for solving the problem is to separate the ring for the momentum correction and the RI storage ring. For this proposal, the RI after the momentum correction in the first storage ring is injected into the second accumulator ring, and the momentum correction in the cooler ring can be operated without the interference to the RI accumulation in the storage ring.

The momentum correction through the single path in an injection line or multiturn operations in a storage ring may be selected by the situation, such as the initial particle energy, pulse duration, and limitation of maximum voltage applied by the induction module. The scheme using multiturn momentum correction in a ring can considerably reduce the requirement of the voltage level and its accuracy tolerance. Since the cooling time of this proposed scheme is very fast, it can be expected to be a useful tool for 
experiments with short-lived nuclei and/or a large number of accumulated RIs.

\section{ACKNOWLEDGMENTS}

We thank Dr. A. Ozawa, Dr. M. Okamura, Dr. Y. Shimosaki, Dr. N. Fukunishi, and Dr. M. Wakasugi for helpful discussions. This work was partly supported by JSPS (Japan Society for the Promotion of Science) and MEXT (Ministry of Education, Culture, Sports, Science and Technology).

[1] I. Tanihata, H. Hamagaki, O. Hashimoto, Y. Shida, N. Yoshikawa, K. Sugimoto, O. Yamakawa, T. Kobayashi, and N. Takahashi, Phys. Rev. Lett. 55, 2676 (1985).

[2] P. Schmor, in Proceedings of the 2003 Particle Accelerator Conference, Oregon (IEEE, Piscataway, NJ, 2003), p. 6.

[3] H.-J. Kluge, K. Blaum, and C. Scheidenberger, Nucl. Instrum. Methods Phys. Res., Sect. A 532, 48 (2004).

[4] B. Franzke, P. Beller, K. Beckert, A. Dolinskii, P. Hulsmann, F. Nolden, C. Peschke, and M. Steck, Nucl. Instrum. Methods Phys. Res., Sect. A 532, 97 (2004).

[5] T. Radon et al., Phys. Rev. Lett. 78, 4701 (1997).

[6] J. Stadlmann et al., Phys. Lett. B 586, 27 (2004).

[7] Y. Yano, A. Goto, M. Kase, and T. Katayama, in Proceedings of the 2001 Particle Accelerator Conference, Chicago (IEEE, Piscataway, NJ, 2001), p. 575 .

[8] T. Kubo, Nucl. Instrum. Methods Phys. Res., Sect. B 204, 97 (2003).

[9] I. Meshkov, W. Mitting, P. Roussel-Chomaz, A. Sidorin, A. Smirnov, and E. Syresin, Report No. GANIL-R-200204, 2002.
[10] I. Meshkov, W. Mitting, P. Roussel-Chomaz, A. Sidorin, A. Smirnov, and E. Syresin, Nucl. Instrum. Methods Phys. Res., Sect. A 523, 262 (2004).

[11] I. N. Meshkov, A.O. Sidorin, A. V. Smirnov, E. M. Syresin, I. V. Titkova, W. Mittig, and P. RousselChomaz, At. Energ. 94, 18 (2003).

[12] N. Inabe, K. Yoshida, M. Wakasugi, and T. Katayama, in Proceedings of the 6th European Particle Accelerator Conference, Stockholm (Institute of Physics, Bristol, 1998), p. 1037.

[13] M. Beutelspacher, H. Fadil, T. Furukawa, M. Grieser, A. Noda, K. Noda, D. Schwalm, T. Shirai, and A. Wolf, Nucl. Instrum. Methods Phys. Res., Sect. A 532, 123 (2004).

[14] K. Horioka et al., Nucl. Instrum. Methods Phys. Res., Sect. A 415, 291 (1998).

[15] T. C. Sangstar et al., Nucl. Instrum. Methods Phys. Res., Sect. A 415, 310 (1998).

[16] K. Takayama, J. Kishiro, M. Sakuda, Y. Shimosaki, and M. Wake, Phys. Rev. Lett. 88, 144801 (2002).

[17] Y. Shimosaki, E. Nakamura, K. Takayama, K. Torikai, M. Watanabe, M. Nakajima, and K. Horioka, Phys. Rev. ST Accel. Beams 7, 014201 (2004).

[18] M. Watanabe, M. Nakajima, M. Shiho, K. Horioka, K. Takayama, and J. Kishiro, Rev. Sci. Instrum. 73, 1756 (2002).

[19] K. Horioka, M. Nakajima, M. Watanabe, M. Honda, E. Hotta, M. Shiho, M. Ogawa, J. Hasegawa, J. Kishiro, and K. Takayama, Laser Part. Beams 20, 609 (2002).

[20] H. Kumagai, A. Ozawa, N. Fukuda, K. Summerer, and I. Tanihata, Nucl. Instrum. Methods Phys. Res., Sect. A 470, 562 (2001).

[21] M. Matsumoto and T. Nishimura, ACM Trans. Model. Comput. Simul. 8, 3 (1998).

[22] C. H. Kim and L. Smith, Part. Accel. 18, 101 (1985).

[23] W. R. Meier, J. J. Barnard, and R. O. Bangerter, Nucl. Instrum. Methods Phys. Res., Sect. A 464, 433 (2001). 